\title{
A qualitative study of barriers to the registration of patients' social histories
}

\author{
Annemarie Dencker ${ }^{1}$, Tine Tjørnhøj-Thomsen ${ }^{1}$, Susanne O Dalton ${ }^{2}$, and Pia Vivian \\ Pedersen $^{1}$ \\ ${ }^{1}$ University of Southern Denmark \\ ${ }^{2}$ Danish Cancer Society Research Center
}

January 7, 2021

\begin{abstract}
Objective All cancer sites show considerable social inequality in risk and patient survival. Despite the advantages of healthcare professionals (HPs) systematically registering information about patients' social circumstances, this is not routine practice. Our aim was to understand the barriers to registering patients' social histories. Methods We observed 104 outpatient encounters and interviewed $30 \mathrm{HPs}$ treating malignant melanomas, urological cancer or lung cancer. We explored interactive (e.g. processes and activities) and interpretative (e.g. perspectives and meanings) aspects of barriers to recording patients' social data. Using Kirk's theoretical framework developed in hospital settings, we conducted a qualitative analysis to address organizational and professional barriers. Results Unevenness in registration was due to 1) organizational barriers (interdisciplinarity, time and technical challenges), 2) professional barriers (prioritization, meeting patients where they are, and reliance on HP's own judgements). HPs neglected to use medical records, preferring to form their own pictures of patients' needs to avoid prejudices and to promote equality. However, HPs did not systematically verify these impressions with the patients. Conclusion To facilitate systematic recording of patients' social histories and address the barriers identified in our study, there is a need to: 1) develop a electronical tool for recording patients' social circumstances that is aligned with HP's daily practices 2) introduce training programmes targeting all barriers, and 3) provide time and opportunities to support patients.
\end{abstract}

\section{A qualitative study of barriers to the registration of patients' social histories Objective}

All cancer sites show considerable social inequality in risk and patient survival. Despite the advantages of healthcare professionals (HPs) systematically registering information about patients' social circumstances, this is not routine practice. Our aim was to understand the barriers to registering patients' social histories.

\section{Methods}

We observed 104 outpatient encounters and interviewed 30 HPs treating malignant melanomas, urological cancer or lung cancer. We exploredinteractive (e.g. processes and activities) andinterpretative (e.g. perspectives and meanings) aspects of barriers to recording patients' social data. Using Kirk's theoretical framework developed in hospital settings, we conducted a qualitative analysis to address organizational and professional barriers.

\section{Results}

Unevenness in registration was due to 1) organizational barriers (interdisciplinarity, time and technical challenges), 2) professional barriers (prioritization, meeting patients where they are, and reliance on HP's own judgements). HPs neglected to use medical records, preferring to form their own pictures of patients' needs 
to avoid prejudices and to promote equality. However, HPs did not systematically verify these impressions with the patients.

\section{Conclusion}

To facilitate systematic recording of patients' social histories and address the barriers identified in our study, there is a need to: 1) develop a electronical tool for recording patients' social circumstances that is aligned with HP's daily practices 2) introduce training programmes targeting all barriers, and 3) provide time and opportunities to support patients.

\section{Questions to be answered}

\section{What is already known about this topic?}

While social inequality in cancer has been documented over the past decade or so, knowledge about patients' social circumstances (e.g. employment and family relationships), social network, educational background, health literacy and mental wellbeing is not routinely assessed in medical practice. This is a problem, since research points to the advantages of healthcare professionals (HPs) systematically registering patients' social data and using the information when communicating with them about their treatment.

What does this article add?

The present study adds a detailed insight into organizational and professional barriers associated with recording patients' social circumstances. This is important because using this information can facilitate patients' access to the help and support they need to get the best out of their cancer treatment.

\section{Background}

Across all cancer sites, risk and survival are reflected in social inequalities, observed as differences in stage at diagnosis and in access to treatment and rehabilitation and in survivorship relative to socio-economic position $(1,2,3,4)$. While this general picture of social inequality in cancer has been documented over the past decade or so, knowledge about patients' social circumstances (e.g. employment, housing condition and family relationships), social network, educational background, health literacy and mental wellbeing is not routinely assessed in medical practice $(5,6,7,8)$.

This is a problem, since research points to the advantages of healthcare professionals (HPs) systematically registering patients' social data and using the information when communicating with them about their treatment. According to Adler \& Stead 2015 and Diez Roux et al 2015 (9,10), assessing information on education, social network and physical activity leads to a) greater precision in diagnosis and treatment through reduction of bias and improved risk stratification, b) more effective decisions, because clinicians suggest more informed and realistic treatment, c) improved detection of social or behavioural risk factors, and d) improvement in care and speed of recovery. Finally, knowledge about patients' social circumstances also allows referrals to relevant services in cases of social isolation, malnutrition $(10,11)$, for financial counselling (9) and helps in managing chronic conditions such as diabetes (9). Despite these important findings, knowledge about patients' social background is not systematically made available.

Our study therefore aimed to explore HP's barriers to recording patients' social history. In our theoretical considerations we follow Kirk et al. (12), who distinguish three levels of barrier as regards mobilization practices in hospital settings: organizational, professional and individual. Organizational barriers might involve shortages of staff and time or issues of teamwork and communication; professional barriers to perceptions of HP's professional identity understood as 'a profession's way of thinking, language codes, explanatory forms and routines of action and what they consider to be their core tasks' (12, p. 2); individual barriers to HP's doubts and fears concerning their professional tasks (12). In the context of our study, we address professional values rather than HP's individual feelings and have chosen to highlight the category of professional barriers.

\section{Methods}


We conducted 30 semi-structured interviews with 15 doctors and 15 nurses and observations of 104 encounters treating malign melanoma (38 encounters), urological cancer (36 encounters) and lung cancer (30 encounters) from May 2019 to October 2019. We explored HPs' barriers to registering patients' social data in the medical record, including structural conditions, practices and attitudes. We chose these treatment groups to reflect differences in socio-economic distribution (1).

\section{Participants}

We recruited doctors and nurses through the management on the three wards. We sought an equal distribution across age, gender and medical specialization. Doctors and nurses gave their informed written consent before participating. All patients and companions, who attended the outpatient clinic, received information about the aims and procedure of our study. Having given informed written consent to participate, patients and companions filled out a short questionnaire giving their gender, age, years of education, occupation and civil status. All consented to participate except from two patients who did not want to participate for private reasons. The first author timed all encounters. The encounters lasted 22 minutes on average (ranging from 7 to 60 minutes): malign melanoma 25 minutes, urological cancer 18 minutes and lung cancer 22 minutes.

Table 1 and 2 describe demographic characteristics for HPs, patients and companions respectively. We did not interview patients and companions, but they participated in the encounters observed.

\section{Procedures}

Interviews were divided equally across the three wards ( 5 doctors and 5 nurses on each ward). We also observed interprofessional collaborations and conferences during our 15 days of observation.

We had conversations $(13,14)$ with HPs before and after observing their encounters with patients to explore the influence of environmental context on their judgments and practices in 'real time'. Observations were followed up by semi-structured individual interviews $(15,16)$. By combining observations and interviews we captured bothinteractive and interpretative aspects behind barriers for maintaining patients' social histories $(15,17,18,19,20,21)$.

Individual interviews were recorded digitally and transcribed verbatim. Fieldnotes were written during observations and followed up after each day of participant observation. The first author kept a logbook to ensure transparency of the research process, which is crucial for study quality (16).

\section{Data analysis}

The process of data analysis was recursive and dynamic and intensified after data collection had ended (22). During observations and semi-structured interviews, emerging analytical ideas and methodological adjustments were noted in the logbook.

We coded the data, grouping codes into general categories and overall themes thereby capturing recurrent patterns across the data $(12,22,23,24)$. In this process, we followed Kirk and found, for example, that: 'interprofessional obstacles', 'lack of time', and 'technical issues' emerged as themes indicating organizational barriers. At the professional level, we identified the themes 'low prioritization', 'meeting patients where they are', and 'reliance on HPs own impressions'. We also found that HPs perceived 'avoiding prejudices' and 'promoting equality' as important professional values.

\section{Results:}

Observation of HP's recording of social data showed up its inadequacies. In the following, we present the results. Having accounted for the arbitrary nature of HP's registration of patients' social histories we outline the organizational and professional barriers to recording patients' social circumstances. We use D in citations for 'doctors' and $\mathrm{N}$ for 'nurses'. Table three provides an overview of themes and subthemes.

\section{Arbitrary registration}


During observations, we examined HP's prior knowledge about patients' social background. If HPs, especially doctors, did register social data, they did not do so systematically and seldom knew about patients' social status or background. Furthermore, if asked about a patient's social background, doctors were often unable to find information in the medical record since it might not have been entered. Moreover, if the medical record included social data, it was rarely precise. For example, records might describe patients as a 'senior citizen' (fieldnotes) without specifying the nature of their profession.

Doctors and nurses registered medical information about patients in their respective digital records, often 'visiting' them to check medical information. While they claimed to prefer sharing social information about patients at medical conferences, observation showed that this was not routine. If patients' social circumstances were brought up, this mainly occurred in extraordinary cases such as severe alcohol abuse, homelessness or lack of compliance.

Nonetheless, the medical record did usually indicate whether the patient or the nearest companions were qualified healthcare professionals, suggesting a need to be extra well-prepared to answer any medical question. HPs sometimes searched for social information in the medical record if communication with a patient was difficult, hoping that this would facilitate communication. However, attempts to record and access information about patients' social circumstances were unstructured.

\section{Organizational barriers}

We identified the following organizational issues as causing the most significant barriers: (1) interprofessional collaboration, (2) shortage of time and support possibilities, and (3) technical challenges.

\section{Interprofessional collaboration}

While doctors only registered social information sporadically, nurses more often registered the patient's social history on arrival:

"It is mostly something that the nurses take care of. They know more about the social background. But the communication between the doctors and the nurses is not always optimal [laughs]. So, it is possible that something gets lost" (D5:2).

Discussion of patients' social circumstances required doctors and nurses to share information, especially since doctors considered this mainly a task for nurses.

Some HPs found that interprofessional collaboration between doctors and nurses was limited due to cuts, leading to a reduction of information exchange about patients' social circumstances.

Exchange of social information about patients also depended on the nurses' ability to put patients' social circumstances on the agenda. Some younger nurses felt they lack the authority to introduce social issues:

"When you sit with the doctor in the outpatient clinic, it is mainly the doctor who speaks with the patient and then you must be quite good as a nurse to address social issues that might be relevant for the patient... [T]here are some nurses who are tougher and dare to enter the conversation (N2:3).

Our observations from outpatient clinics showed that doctors typically set the agenda for patient encounters, nurses playing a supportive role. The tone adopted in interprofessional communication often determined the extent to which nurses interfered or offered information about the patients' social circumstances.

\section{Lack of time and support possibilities}

Despite nurses asking more about patients' social background than doctors, they felt unable to register social information adequately since competing medical information had to be recorded within certain time limits:

"And we really do try to document everything [in the medical record], but I just feel we drown in it". (N3:3).

Lack of time reduced HP's primary focus to handling immediate situations in their daily work: "Because of the system [time] we are forced to walk with blinkers" (D1:5). HPs described how doctors previously dictated 
information about patients, they now had to write patients' records themselves. Pressure of time meant entering only essential information - which did not include social information unless it affected immediate treatment. HPs would characterize social information as 'other stuff':

"It is also about getting through the day, and you don't have so much time for other stuff." (D5:2).

Unsure how to respond or where to find support, lack of time led HPs to avoid exploring patients' difficulties.

Shortage of time also led HPs to rely on their own observations of the patients' needs rather than asking the patients and recording information based on the patients' own words.

"But time is just so pressurized that it is hard to do it[writing in the medical record] systematically. It is something you must sense with the patients" (D1:2).

\section{Technical challenges}

Both doctors and nurses found that electronic recording was troublesome and reductionistic, since they had to complete pre-coded sentences, which often did not match the nuances of the patient's situation:

"It is pre-coded. Earlier you could make it a bit more personal. So, it becomes too template-like sometimes."(D3.1).

HPs found that the medical record system prevented them from documenting social information adequately because keywords had to match the template. HPs explained that entering detailed information about patients' social circumstances required more clicks in the electronic system. Consequently, they would refrain from adding details, especially when extra time was spent on electronic delays.

\section{Professional barriers}

The following professional barriers were found to be the most significant: (1) low prioritization, (2) meeting patients where they are, and (3) reliance on HP's own impressions.

\section{Low prioritization}

Nurses may often have recorded patients' social circumstances in the beginning of an illness trajectory, but neither nurses nor doctors perceived maintaining patients' social histories as their main task. Patients' social data did not carry the significance of medical data:

"I don't think we think about social differences at all because our main focus is on the illness." (N4:1).

Patients' social circumstances did, however, have particular relevance on specific wards. Patients on the lung cancer and the urological wards could expect many side-effects for longer periods. Here, HPs seemed more dependent on understanding patients' capacity to comply with treatment, including social information about support in their everyday lives.

Despite nurses' intentions on all three wards to ask patients about their social circumstances, any information was not systematically followed up by doctors during the illness trajectory. HPs expressed a preference for encountering the patients in the here-and-now rather than seeking information in their medical record.

\section{Meeting patients where they are}

During interviews, doctors and nurses alike frequently used the expression 'meeting patients where they are'. Knowing about patients' social circumstances prior to this meeting was not considered useful or relevant to communicating:

"I don't know what people have been doing [jobwise].I do not know about their education. I hardly care about it. I treat the patients for what they come for and then we take it from there. I meet the patient, where the patient is." (D2:1)

Meeting patients 'where they are' also meant being open to what might happen in the situation: 
"I also think it is a problem if you look through the diagnostic list and decide how to talk with the patient instead of being open to what happens along the way" (D1:1).

HPs explained that other existential factors such as responses to crises could prove significant and were independent of a patient's social background:

"You may have information about the patient in advance, but you must meet the person where the person is. Many patients here are in crisis and then you must, of course, take stock of the situation"(D5:1).

'Taking stock of the situation' meant forming an overall impression of the patient here and now, which might mean judging that the patient was emotionally overwhelmed and adjusting information accordingly.

HPs were frequently surprised by patient reactions and outcomes. A patient could appear well-educated, well-off, physically strong and in every way prepared to start treatment. And then, they could suddenly break down mentally, and prove to have family or alcohol problems. Conversely, there were patients with little education and poor language, smelling of smoke, who turned out determined and adherent throughout their treatment, successfully supported by close family members. This element of surprise meant that doctors and nurse did not find prior knowledge of the patients' social circumstances essential:

"It is a nice information to have where people live and what they do, but you can't use it, if you haven't met the man" (D1:3).

HPs felt it important not to prejudge patients by accessing information about their social circumstances. They explained that they wanted to address the patient as an individual, not as a category:

"I mostly think of the patient as an individual who enters the door, and then I have to find out where this particular person is, and what I should contribute, and then I . . try to see the patient as an individual instead of a social category" (N3:2).

HPs prioritized their own observations, concerned that categorization might lead to unequal treatment:

"Actually, I don't think that patients' social position means so much. Because I think we should meet the patients equally" (N4:3).

This meant 'taking stock', 'meeting patients where they were' and avoiding social stereotyping.

\section{Reliance on HPs own impressions}

To meet patients 'where they are', HPs relied on their own impressions of their preferences and needs and not on factual knowledge from the medical records. For example, there were some patients who smelled of urine or smoke, had long nails, puffy skin, or greasy hair or were dressed in dirty, crumpled clothes. When HPs encountered such patients, they tried to pay special attention to them by asking them how they were doing or reminding them of their next appointment. HPs tried not to plan consultations too early in the morning to increase the probability of patients showing up. When patients had poor hygiene, HPs also tried to simplify information:

"If I sense that the patient has social challenges,... then I don't use technical terms and I don't go into too much detail. Then I give a general description, and if they have elaborate questions, then I answer them of course" (D3:1).

HPs preferred to use their own impressions in targeting their communication and their information:

"I try to talk with the patient, so it matches how I sense his intellectual level" (D2:1).

Finding out 'where the patient is' was also facilitated by listening to the questions patients asked. HPs explained that patients' questions could reflect their understanding of their illness and treatment. HPs also listened to the patients' language, to the level of detail and complexity in presenting their problem, and tried to adapt their information accordingly: 
"Some patients quote the medical record using the right words. Others just say: I had a spot on my skin with something in it. So, it is about finding out where to start" (D5:1).

We observed that the more detailed the patients' description of their problems, the more detailed was information they were given. Those patients who arrived with detailed notes about their condition and questions for the HPs, especially those who brought a companion, were given more in-depth information about their illness and treatment than patients who showed up alone and with no visible preparation.

\section{Discussion}

The present study provides a detailed insight into barriers associated with recording patients' social circumstances. This is important because using this information can facilitate patients' access to the help and support they need to get the best out of their cancer treatment. Drawing on Kirk's framework of barriers, we explored and identified both organizational and professional barriers to maintaining patients' social histories.

We found that HPs preferred to form their own impression of patients rather than reading about them beforehand in the medical records. It was important for them to sustain a situational, individualized, openminded approach to patients in order to treat them all equal respect and without prejudice. These good intentions may, however, have the opposite effect, if patients are not asked whether the HP's ideas about them are in accordance with the patients' own experiences. This disparity becomes critical when dealing with patients who do not proactively express their needs themselves. Several studies show that less socially adapted patients are less likely to share their experiences, to ask questions and to involve themselves actively in the treatment $(25,26)$.

For example, a qualitative study that included 58 oncologists and 405 patients showed that younger patients, those who were white, had more than a high school education and reported high or medium income, asked and were asked more questions by physicians than non-white, less educated and less affluent patients. Further, physicians spent more time engaging in relationship-building with the socially most advantaged patients (25). The study suggests that physicians themselves are not aware of these tendencies, which might lead to disparities in patient outcomes (ibid). Another qualitative study including 73 men and women showed that more highly educated patients are more likely to perceive their involvement in healthcare decision-making as sharing the responsibility with the doctor, actively relating to and exploring information they receive. Less well-educated patients tended to construct their involvement in a more passive way, consenting or not consenting to recommendations presented by the doctor (26). If, therefore, HPs do not elicit patients' own viewpoints but only trust their own impressions, they do not get to understand how patients' social circumstances might influence the treatment of their disease.

We also found that organizational barriers reinforced professional barriers. So, when time was short, HPs tended to prioritize their own impressions over questioning the patient and recording their experiences and needs. Lack of time also led to a prioritization of medical information above social information. That said, HP's situational approach to patient communication - articulated as 'meeting patients where they are' seemed to be well-founded and provided professional value to HPs that might well co-exist with registering of patients' social histories.

\section{Clinical Implications}

While more data is needed to make clinical recommendations regarding barriers to HP's recording and use of patient social histories, this particular study would support 1) the development of a electronical tool for recording patients' social data that is aligned with HP's daily practices, 2) training programmes targeting all barriers, including HP's awareness of their own assumptions about patients, and 3) allowing time and opportunities to support patients who need it.

\section{Study Limitations}

Our study builds on many qualitative, semi-structured, in-depth interviews and out-patient clinic observations. Combining observation and interviews was important in revealing barriers to recording patients' social 
circumstances. However, our study presents some limitations.

Firstly, we did not explore patients' perspectives. This can be problematic because patients' perspectives might not match our preconceptions.

Secondly, we have not explored whether there might be essential differences between wards that influence the relevance - and importance - of recording social data in different ways. The best ways of maintaining patients' social histories might be related to factors peculiar to a specific ward.

Thirdly, this study is based on the idea that patient social histories are a 'good thing'. However, knowing a patient's job or age cannot stand on its own, since objective descriptions conceal individual variety. Thus, the future might benefit from a 'two-handed' approach rather than an 'either/or', finding a dynamic balance between recording social histories and sensing the patient in the given situation.

\section{Conclusion}

We found that various barriers impede the systematic recording of patients' social histories. HPs tend to rely on their own impressions of patients' abilities, needs and wishes without necessarily involving the patients by checking these impressions. In practice, this might lead to some patients getting less support and detailed information than they might have wished, and to HPs missing important information about difficulties patients face - and about their resources - while undergoing cancer treatment. Future studies might investigate the effects of systematic recording of social histories on treatment outcomes.

\section{Ethics and number}

We have followed basic rules for ethical conduct in qualitative research, and the study was approved by the University of Southern Denmark in accordance with the European GDPR legislation. Registration number: J.nr. 2019-40.413.

\section{Data availability}

Due to the European GDPR legislation, data can only be shared between relevant researchers and not made available to the public or any other non-employee of University of Southern Denmark.

\section{Acknowledgements}

We are grateful to the Danish Cancer Society National Center for Optimal Outcomes for All (COMPAS) for funding this research.

\section{Conflict of interest}

The authors have no conflict of interest to declare.

\section{References}

1. Dalton SO, Schuz J, Engholm G, et al. Social inequality in incidence of and survival from cancer in a population-based study in Denmark, 1994-2003: Summary of findings. Eur. J. Cancer 2008; 44(14): 2074-85.

2. Dalton SO, Olsen MH, Johansen C, Olsen JH, Andersen KK. Socioeconomic inequality in cancer survival - changes over time. A population-based study, Denmark, 1987-2013. Acta Oncol 2019; 58 (5): 737-744.

3. Nordahl H, Lange T, Osler M, et al. Education and cause-specific mortality: the mediating role of differential exposure and vulnerability to behavioral risk factors. J Epidemiol 2014; 25(3 PG - 389-96): 389-96.

4. Søgaard M, Thomsen RW, Bossen KS, Sorensen HT, Norgaard M. The impact of comorbidity on cancer survival: a review. Clin. Epidemiol. 2013; 5(Suppl 1): 3-29.

5. Giuse NB et al. Institute of Medicine Measures of Social and Behavioral Determinants of Health: A Feasibility Study. Am J Prev Med 2017; 52(2): 199-206. 
6. Uitterhoeve RJ, Bensing JM, Grol RP, Demulder PH, Van Achterberg T. The effect of communication skills training on patient outcomes in cancer care: a systematic review of the literature. Eur J Cancer Care 2010; 19: 442-457.

7. Riiskjær E. Patienten som partner: En nødvendig idé med ringe plads. [The patient as a partner. A necessary idea with little traction] Odense, Denmark: Syddansk Universitetsforlag; 2014.

8. Schouten B et al. Healthcare professionals' perspectives on the prevalence, barriers and management of psychosocial issues in cancer care: A mixed methods study. Eur J Cancer Care 2018. Jan; 28(1):e12936. doi: 10.1111/ecc.12936. Epub 2018 Oct 9.

9. Adler NE, Stead WW. Patients in Context - ERH Capture of Social and Behavioral Determinants of Health. N. Engl. J. Med. 2015; 372(8): 698-701.

10. Diez Roux et al. Social and Behavioral Information in Electronic Health Records New opportunities for Medicine and Public Health. Am J Prev Med 2015; 49 (6): 980-983.

11. Arends J. Struggling with nutrition in patients with advanced cancer: Nutrition and nourishment focusing on metabolism and supportive care. Ann. Oncol. 2018; 29 (22): 27-34.

12. Kirk JW et al. Disentangling the complexity of mobility of older medical patients in routine practice: An ethnographic study in Denmark. PLoS One 2019, 14 (4): 1.24. DOI:10.1371/journal.pone.0214271.

13. Carpiano RM. Come take a walk with me: The "Go-Along" interview as a novel methods for studying the implications of place and health and well-being. Health Place 2009; 15:263-72.

14. Garcia CM, Eisenberg ME, Frerich EA, Lechner KE, Lust K. Conducting go-along interviews to understand context and promote health. Qual Health Res 2012; 22:1395-1403.

15. Rubin HJ, Rubin IS. Qualitative interviewing. The art of hearing data. London: Sage Publications; 2005.

16. Tjørnhøj-Thomsen T, Whyte SR. Fieldwork and Participant Observation. I Research Methods in Public Health, eds Vallgårda S \& Koch L. København: Gyldendal Akademisk; 2008.

17. Brewer JD. Etnography. Buckingham: Open University Press; 2000.

18. Grills S. An Invitation to the Field. Fieldwork and the Pragmatists' Lesson. In: S. Grills (ed.) Doing Ethnographic Research. Fieldwork Settings. London: SAGE publication; 1998.

19. Madden R. Being ethnographic. A Guide to the Theory and Practice of Ethnography. London: SAGE publication; 2010.

20. Atkinson P. For Etnography. London. SAGE publication; 2015.

21. Shim JK. Cultural Health Capital: A Theoretical Approach to Understanding Health Care Interaction and the Dynamics of Unequal Treatment. J Health Soc Behav 2010; 51:1-15.

22. Merriam SB. Qualitative Research. A Guide to Design and Implementation: San Francisco: Jossey Bass; 2009.

23. Patton MQ, Qualitative Research \& Evaluation Methods. Thousand Oaks. Sage Publications; 2002.

24. Mason J. Organizing and indexing Qualitative Data; In: Mason J.: Qualitative Researching. London: SAGE Publications; 2007.

25. Siminoff LA, Graham GC, Gordon NH. Cancer communication patterns and the influence of patient characteristics: Disparities in information-giving and affective behaviors. Patient Educ Couns 2006; 62: 355-360.

26. Smith SK et al. Exploring patient involvement in healthcare decision making across different education and functional health literacy groups. Soc. Sci. Med. 2009, 69: 1805-1812.

Table 1: Characteristics of doctors and nurses $(n=30)$

\begin{tabular}{lll}
\hline & Doctors $(\mathbf{n}=\mathbf{1 5})$ & Nurses $(\mathbf{n}=\mathbf{1 5})$ \\
\hline Gender & & \\
Male & 5 & 0 \\
Female & 10 & 15 \\
Age & $29-60$ (mean: 42$)$ & $28-66$ (mean: 45) \\
Years of experience & $2-32($ mean: 14$)$ & $1-44$ (mean: 19) \\
\hline
\end{tabular}


Table 2: Characteristics of patients and companions $(n=169)$

\begin{tabular}{lll}
\hline & Patients $(\mathbf{n}=\mathbf{1 0 4})$ & Companions $(\mathbf{n}=\mathbf{6 5})$ \\
\hline Gender & & \\
Male & 60 & 21 \\
Female & 44 & 44 \\
Education & & \\
Short (7-13 years) & 33 & 23 \\
Medium (14-16 years) & 37 & 26 \\
Long (17+) & 21 & 16 \\
Age & & \\
$<40$ & 19 & 11 \\
$40-60$ & 19 & 22 \\
$60-80$ & 56 & 29 \\
$>80$ & 10 & 3 \\
Ward & & \\
Lung cancer & 30 & 24 \\
Urological cancer & 36 & 17 \\
Malign melanoma & 38 & 24 \\
Civil status & & \\
Single & 24 & 6 \\
In a relationship & 80 & 59 \\
Brought at least one companion & & \\
Yes & 48 & - \\
No & 56 & - \\
Type of companion & & 44 \\
Spouse & - & 15 \\
Adult children & - & 4 \\
Parents & - & \\
Friends or other family & - & \\
\hline
\end{tabular}

\section{Hosted file}

Table 1.pdf available at https://authorea.com/users/387942/articles/502812-a-qualitativestudy-of-barriers-to-the-registration-of-patients-social-histories

\section{Hosted file}

Table 2.pdf available at https://authorea.com/users/387942/articles/502812-a-qualitativestudy-of-barriers-to-the-registration-of-patients-social-histories

\section{Hosted file}

Table 3.pdf available at https://authorea.com/users/387942/articles/502812-a-qualitativestudy-of-barriers-to-the-registration-of-patients-social-histories 